\title{
Oil spill detection from SAR image using SVM based classification
}

\author{
A. A. Matkan, M. Hajeb, Z. Azarakhsh* \\ Remote Sensing \& GIS Department, Shahid Beheshti University, Evin, Tehran, Iran. (a-matkan, \\ M_Hajeb)@sbu.ac.ir, zi.azarakhsh@Gmail.com
}

KEY WORDS: oil spill, SVM, Decomposition, POLSAR, Texture Analysis

\begin{abstract}
:
In this paper, the potential of fully polarimetric L-band SAR data for detecting sea oil spills is investigated using polarimetric decompositions and texture analysis based on SVM classifier. First, power and magnitude measurements of HH and VV polarization modes and, Pauli, Freeman and Krogager decompositions are computed and applied in SVM classifier. Texture analysis is used for identification using SVM method. The texture features i.e. Mean, Variance, Contrast and Dissimilarity from them are then extracted. Experiments are conducted on full polarimetric SAR data acquired from PALSAR sensor of ALOS satellite on August 25, 2006. An accuracy assessment indicated overall accuracy of $78.92 \%$ and $96.46 \%$ for the power measurement of the VV polarization and the Krogager decomposition respectively in first step. But by use of texture analysis the results are improved to $96.44 \%$ and $96.65 \%$ quality for mean of power and magnitude measurements of $\mathrm{HH}$ and VV polarizations and the Krogager decomposition. Results show that the Krogager polarimetric decomposition method has the satisfying result for detection of sea oil spill on the sea surface and the texture analysis presents the good results.
\end{abstract}

\section{Introduction}

In the recent years, oil spill in marine environments is one of the most important factors of marine pollution that has very harmful impact on marine ecosystems, economy and human life. Regarding sea is a valuable source for oil reservoirs and a place for oil extraction and transportation of tankers as well as considering Iran is ended in Caspian sea from north and in Persian gulf and Oman sea in south which are rich sources of oil, rapid and timely identification those pollutions has very much important role in reduction their harmful impacts. Remote sensing technology is an effective way for detecting and operational monitoring of oil spill in the ocean areas (GirardArdhuin et al., 2003; Migliaccio and Trangaglia, 2004; Brekke and Solberg, 2005; Cheng et al., 2011; Zhang et al., 2011).

Previous studies are show which SAR data are suitable for detection of marine oil spill due to their day -and -night and allweather imaging capabilities and having large coverage (Brekke and Solberg, 2005).

Scattering from the ocean's surface is significantly due to scattering from capillary waves and short gravity waves (Fortuny-Guasch, 2003). Oil spills on SAR images damp short gravity-capillary waves and decrease backscatter of sea surface resulting in creating dark formations on SAR images having contrast with surrounding sea surface (Holt, 2004;Brekke and Solberg, 2005; Migliaccio et al., 2007; Fingas and Brown, 2011). This characteristic leads to identification of the spills on SAR images.

The advent of the full-polarimetric satellite SAR systems (i.e. ALOS/PALSAR, RDARSAT2 and TerraSAR-X missions) potentially yield more useful information than single polarization measurements and some recent studies claim that multipolarization data can give better discrimination between oil spills and their surroundings (Gade et al., 1998; Migliaccio and et al., 2007; Topouzelis, 2008, Migliaccio et al., 2009; Velotto et al., 2010).

Information about scattering processes can be obtained from polarimetric SAR (POL-SAR) data by analysing the individual components of the scattering matrix or by using various decomposition methods (Jones et al., 2011). Most decomposition methods attempt to relate the polarimetric backscatter to the physical properties of the scatterers, for example, surface, volumetric, and dihedral scattering (Jones et al., 2011).

A large number of studies aiming at oil spill detection based on SAR images have been conducted (Del Frate et al., 2000; Fiscella et al., 2000; Marghany, 2001; Pavlakis et al., 2001; Karathanassi et al., 2006; Migliaccio and Trangaglia, 2004; Stathakis et al., 2006; Topouzelis et al., 2003, 2009). Some detailed studies to oil spill detection approaches by use of remote sensing data are given by (Brekke and Solberg (2005); Topouzelis (2008); Migliaccio et al. 2010).

There are a limited number of studies using of decomposition methods for oil spill detection. In (Schuler et al., 2004; Migliaccio et al., 2007; Jones et al., 2011; Zhang et al., 2011) the entropy, anisotropy, and average alpha of the Cloude-Pottier decomposition are used for oil-spill detection. polarimetric SAR decomposition parameters, average alpha angle and entropy, are estimated for mapping sea surface oil slicks using a RADARSAT-2 quad-polarization SAR image by (Zhang et al., 2011). Other decompositions such as Shannon entropy decomposition have also been investigated for monitoring and detection of oil spills (Jones et al., 2011).

The main aim in this paper is marine oil spill detection using fully polarimetric SAR data. For this reason, the Pauli, Freeman and Krogager decompositions and texture analysis are used. In this study Support Vector Machine classifier is used for classification.

\section{Materials and methods}

\subsection{Data in used and Study area}

The study area chosen to conduct this paper is related to an oil spill from a sunken tanker near central Philippines in Guimaras Strait. The disaster occurred on August 11, 2006 and this oil spill observed using JAXA reports. Filipino mini-tanker while traveling of Negros Island accidentally sank about $24 \mathrm{~km}$ of the southern coast of Lusaka Point, Guimaras Island of the Philippines, causing heavy oil spill. The PALSAR image of the oil spill area was acquired on August 25. In Figure 1 an RGB Pauli representation of the area is depicted. 
In this paper, oil spills are detected from full polarimetric SAR data of high resolution SAR image of PALSAR sensor of ALOS satellite with approximately $30 \mathrm{~m}$ spatial resolution.
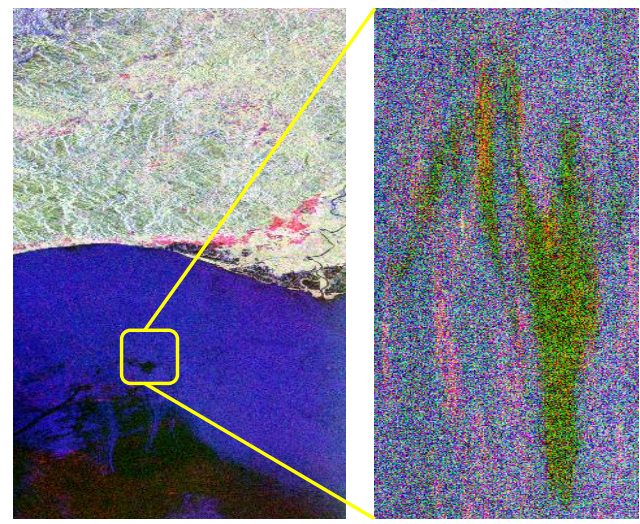

Figure 1. Pauli decomposition of study area

\section{2 methodology}

\subsubsection{The Pauli decomposition}

The Pauli decomposition expresses the measured scattering matrix $[\mathrm{S}]$ in the so-called Pauli basis.

If we considered the conventional orthogonal linear (h, v) basis, in a general case, the Pauli basis is given by the following four $2 \times 2$ matrices (Cloude and pottier, 1996).

$$
\begin{aligned}
& {[S]_{a}=\frac{1}{\sqrt{2}}\left[\begin{array}{ll}
1 & 0 \\
0 & 1
\end{array}\right],[S]_{b}=\frac{1}{\sqrt{2}}\left[\begin{array}{cc}
1 & 0 \\
0 & -1
\end{array}\right]} \\
& {[S]_{c}=\frac{1}{\sqrt{2}}\left[\begin{array}{ll}
0 & 1 \\
1 & 0
\end{array}\right],[S]_{d}=\frac{1}{\sqrt{2}}\left[\begin{array}{cc}
0 & -1 \\
1 & 0
\end{array}\right]}
\end{aligned}
$$

It has been always considered that $\mathrm{Shv}=\mathrm{Svh}$, since reciprocity applies in a monostatic system configuration. In this situation, the Pauli basis can be reduced to a basis composed by the matrices. Consequently, given a measured scattering matrix [S], it can be expressed as follows:

$$
[S]=\left[\begin{array}{ll}
S_{h h} & S_{h v} \\
S_{h v} & S_{v v}
\end{array}\right]=\alpha[S]_{a}+{ }_{\gamma}[S]_{v^{+\beta}}[S]_{b}
$$

Where

$$
\begin{aligned}
& \alpha=\frac{S_{h h}+2 S_{v v}}{\sqrt{2}} \\
& \beta=\frac{S_{h h}-2 S_{v v}}{\sqrt{2}} \\
& \mathrm{y}=\sqrt{2} S_{h v}
\end{aligned}
$$

And it can be easy shown that the span of [S] can be obtained as

$$
\begin{aligned}
& S P A N=\left|S_{h h}\right|^{2}+\left|S_{v v}\right|^{2}+2\left|S_{h v}\right|^{2} \\
& =\alpha^{2}+\beta^{2}+y^{2}
\end{aligned}
$$

\subsubsection{The Krogager decomposition}

Krogager has proposed an alternative to factorize the scattering matrix as the combination of the responses of a sphere, a diplane and a helix. The last two components present an orientation angle $\theta$. If we consider the scattering matrix expressed in the circular polarization basis $(r, l)$, the Krogager decomposition presents the following formulation (Krogager, 1990).

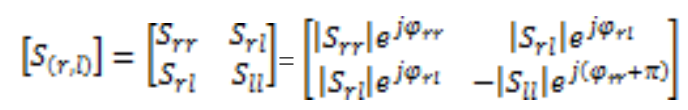

$$
=e^{j \varphi}\left\{e^{j \varphi_{2}} K_{Q}\left[\begin{array}{ll}
0 & j \\
j & 0
\end{array}\right]+K_{d}\left[\begin{array}{cc}
e^{j 2 \theta} & j \\
j & -e^{-j 2 \theta}
\end{array}\right]+K_{h}\left[\begin{array}{cc}
e^{j 2 \theta} & 0 \\
0 & 0
\end{array}\right]\right\}
$$

For the convenience of calculation, the following relations are useful.

$$
\begin{aligned}
& S_{r y}=\left(S_{h h}-S_{V V}+j 2 S_{h V}\right)_{/ 2} \\
& S_{\mathbb{N}}=\left(S_{W D}-S_{h h}+j 2 S_{h v}\right) / 2 \\
& S_{r i}=j\left(S_{h h}+s_{W V}\right) / 2
\end{aligned}
$$

\subsubsection{The Freeman decomposition}

The Freeman decomposition models the covariance matrix as the contribution of three scattering mechanisms (Freeman and Durden, 1992)

- Fv: Volume scattering where a canopy scatterer is modelled as a set of randomly oriented dipoles.

- Fd: Double-bounce scattering modelled by a dihedral corner reflector.

- Fs: Surface or single-bounce scattering modeled by a firstorder Bragg surface scatterer

Where Fs and Fd can be obtained from Equation (9).

$$
\begin{aligned}
& \left|S_{h h}\right|^{2}=f_{s}|\beta|^{2}+f_{d}|\alpha|^{2} \\
& \left|S_{v v}\right|^{2}=f_{s} \\
& S_{h h_{v w}}=f_{s} \beta+f_{d} \beta
\end{aligned}
$$




$$
F v=\left\{\begin{array}{ll}
\left|S_{h h}\right|^{2}+\left|S_{W V}\right|^{2}+2\left|S_{h v}\right|^{2}=\text { span } \\
8\left|S_{h v}\right|^{2} & \left(\left|S_{h h}\right|^{2}<3\left|S_{h v}\right|^{2}\right) \\
8\left|S_{k v}\right|^{2} & \left(\left|S_{W V}\right|^{2}<3\left|S_{h v}\right|^{2}\right)
\end{array}\right\}
$$

\subsubsection{The proposed algorithm}

In this method first, power and magnitude measurements of $\mathrm{HH}$ and VV polarization modes are extracted then the scattering (S), coherency $(\mathrm{T})$ and covariance $(\mathrm{C})$ matrices are constructed from the full polarimetric SAR data to computing the Pauli, Krogager and Freeman decompositions. Afterward, each of them is separately classified by SVM classifier. In the next step, the most popular texture features are extracted from the magnitude and power measurements and the decompositions. The texture features include Mean, Variance, Contrast and Dissimilarity. The mean feature separately and combination of the 4 features (Mean, Variance, Contrast and Dissimilarity) are examined with different window sizes $(3 * 3,5 * 5,7 * 7,9 * 9,11 * 11$ and $17 * 17)$ and an optimum texture feature with the best window size is selected to be used in the classification step. Finally, the result of each of classifications is evaluated by use of comparing with ground truth data obtained by manually digitizing the polarimetric SAR images. The flowchart of proposed algorithm is shown in Figure 2.

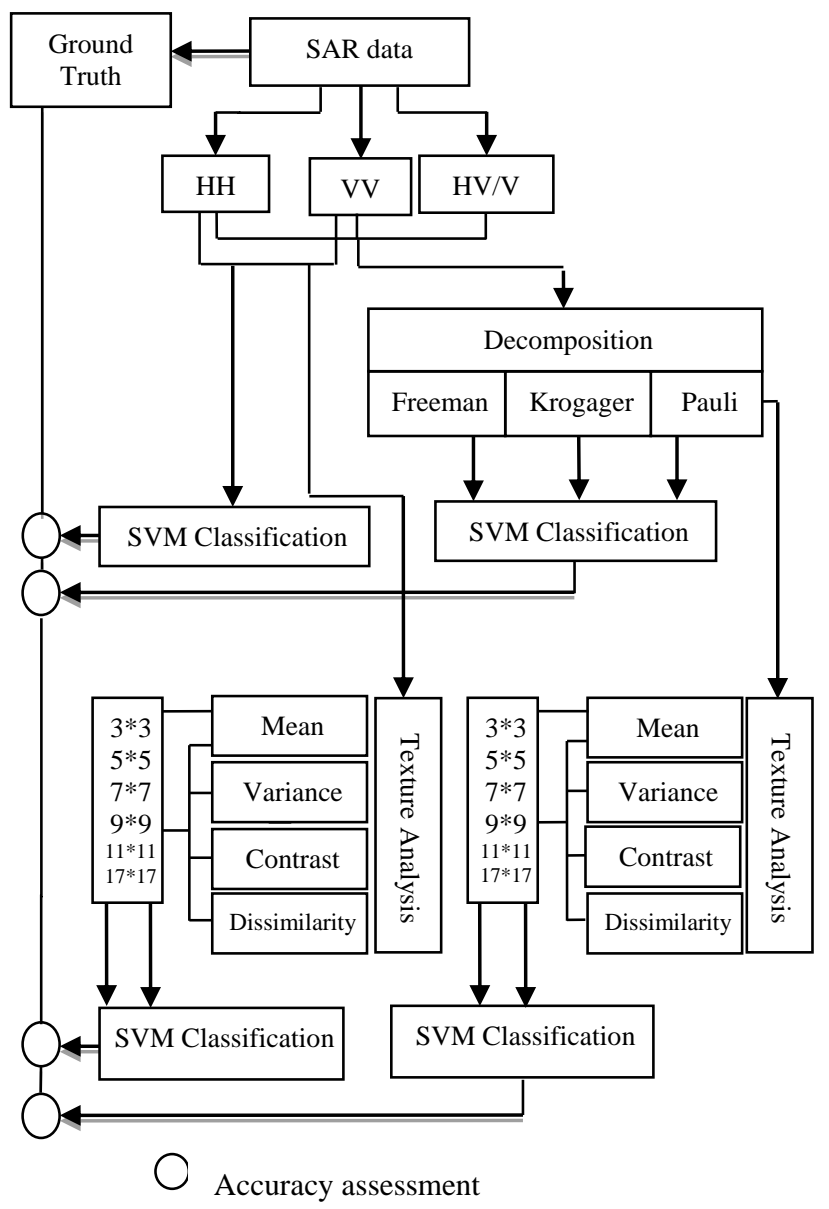

Figure 2. Flowchart of the proposed algorithm

\section{RESULTS AND DISCUSSION}

\subsection{Results}

The algorithm is applied on the fully polarimetric L-band SAR data and the results following:

Magnitude and power measurements derived from $\mathrm{HH}$ and VV polarizations are demonstrated in figure $3 \mathrm{a}, 3 \mathrm{~b}, 3 \mathrm{c}$ and $3 \mathrm{~d}$ respectively. Figures $3 \mathrm{e}$, $3 \mathrm{f}$ and $3 \mathrm{~g}$ show the Pauli, Krogager and Freeman decompositions respectively.

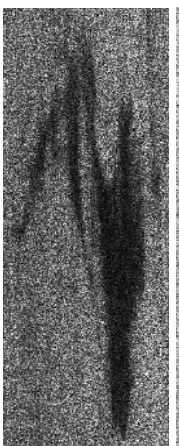

(a)

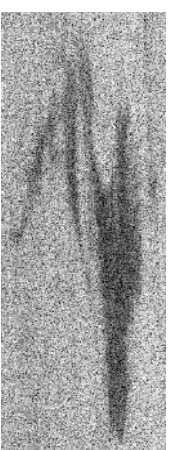

(b)

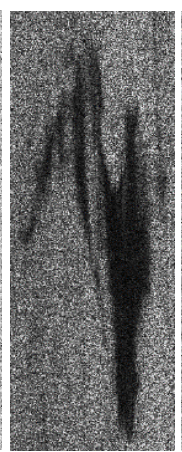

(c)

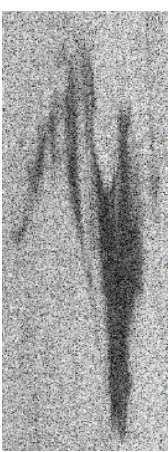

(d)

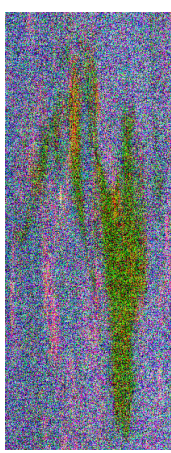

(e)

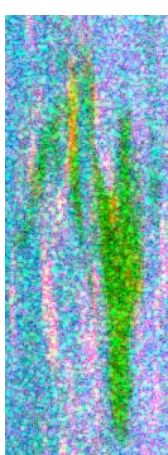

(f)

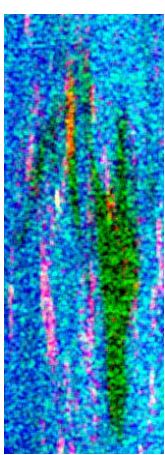

(g)
Figure 3.Interested oil spill images: (a) the magnitude image of HH. (b) The power image of $\mathrm{HH}$ channel. (c) The magnitude image of VV channel. (d) The power image derived from $\mathrm{HH}$ channel. (e) The Pauli decomposition. (f) The Krogager decomposition. (g) The Freeman decomposition.

Magnitude and power measurements of $\mathrm{HH}$ and VV polarizations are classified separately and together. After accuracy assessment it is determined that the power measurement of VV polarization has the best result that is shown in figure 4a. The Pauli decomposition and Krogager and Freeman decompositions with different sizes are separately classified and results depicted in figures $4 \mathrm{~b}, 4 \mathrm{c}$ and $4 \mathrm{~d}$ respectively. Sensitivity analysis of the Krogager and Freeman decomposition window size shows that $5 \times 5$ window is the best. 


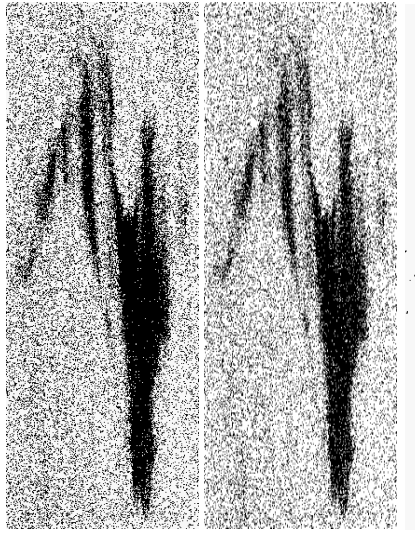

(a)

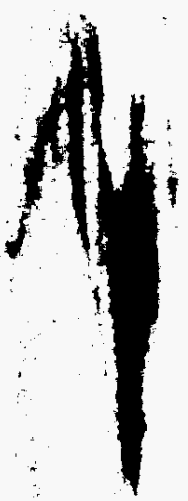

(c)

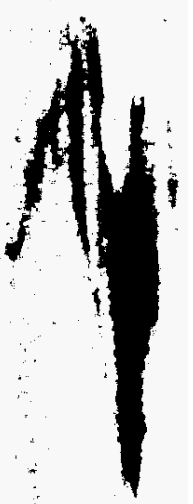

(d)
Figure 4. Results of SVM classification in first step. The classified image obtained from (a) power of $\mathrm{VV}$ polarization. (b) Pauli decomposition. (c) Krogager decomposition. (d) Freeman decomposition

The texture features i.e. Mean, Variance, Contrast and Dissimilarity with window sizes of $3 \times 3,5 \times 5,7 \times 7,9 \times 9,11 \times 11$ and $17 \times 17$ pixels are extracted from the Magnitude and power measurements as well as the Pauli, Krogager and Freeman decompositions. The resulting images are shown in Figure 5.

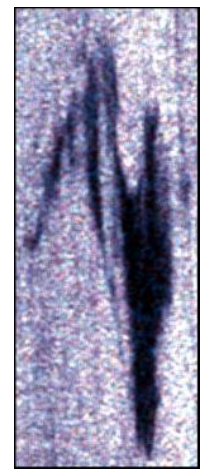

(a)

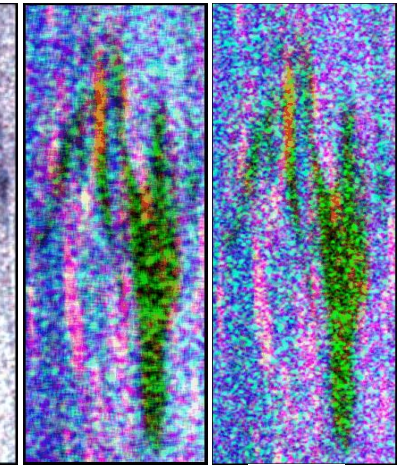

(b)

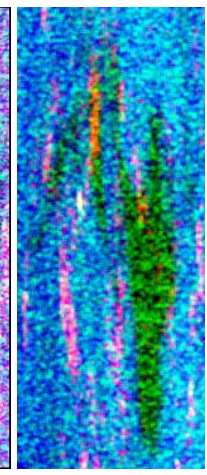

(d)
Figure 5: images of mean of (a) Magnitude and power measures of $\mathrm{HH}$ and VV polarizations and (b) Pauli decomposition. (c) Krogager decomposition. (d) Freeman decomposition in window sizes of $5 \times 5,7 \times 7$, $3 \times 3$ and $5 \times 5$ respectively.

The obtained images from texture analysis of magnitude and power measurements of $\mathrm{HH}$ and VV polarizations and the Pauli, Krogager and Freeman decompositions are classified by SVM classifier. Figure 6 shows the results of the classifications.

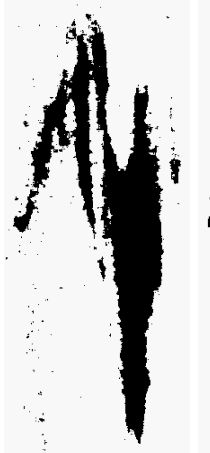

(a)

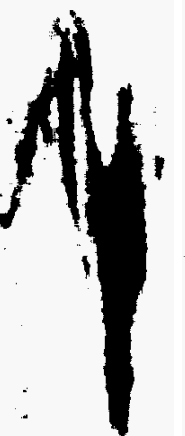

(b)

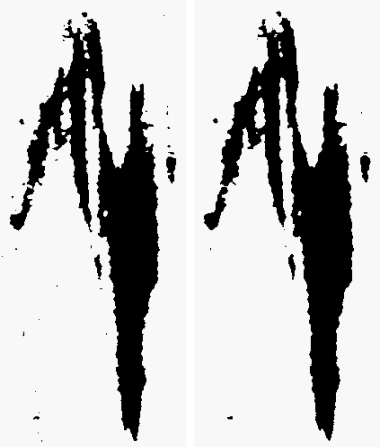

(c)

(d)
Figure6: Resuls of classification of mean of (a) Magnitude and power measurements of $\mathrm{HH}$ and $\mathrm{VV}$ polarizations and (b) Pauli decomposition. (c) Krogager decomposition. (d) Freeman decomposition in window sizes of $5 \times 5,7 \times 7,3 \times 3$ and $5 \times 5$ respectively.

\subsection{Accuracy assessment}

In order to accuracy assessment, the ground truth data are extracted visually and by manually digitizing from the full polarimetric data. The ground truth is shown in Figure 7.

Assessment of obtained results is done by constitution Confusion matrix and computation of overall accuracy and Kappa Coefficient. The Figure 8 shows Overall accuracy of those classifications. Figure 9 and Figure 10 show Overall accuracy of last step classifications respectively.

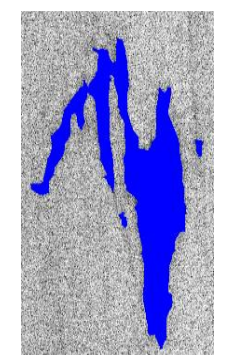

Figure7: Ground truth data imposed on the power image of VV channel

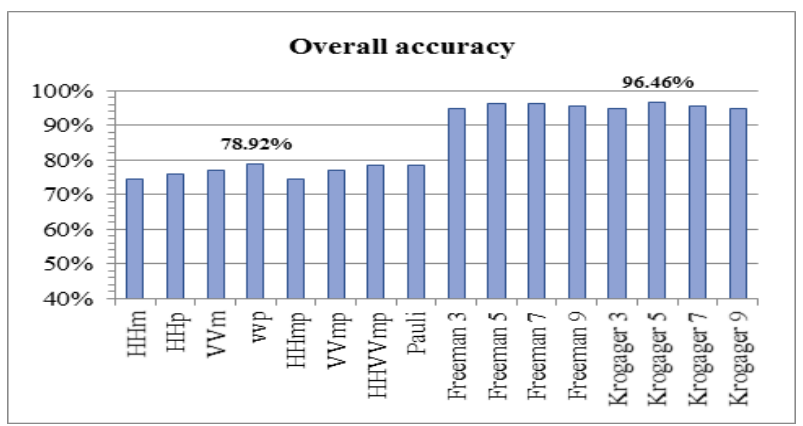

Figure 8. overall accuracy of the SVM classification of the magnitude and power measurements 0 f $\mathrm{HH}$ and $\mathrm{VV}$ channels as well as The Pauli , Krogager and Freeman decompositions. (m: magnitude, p: power) 


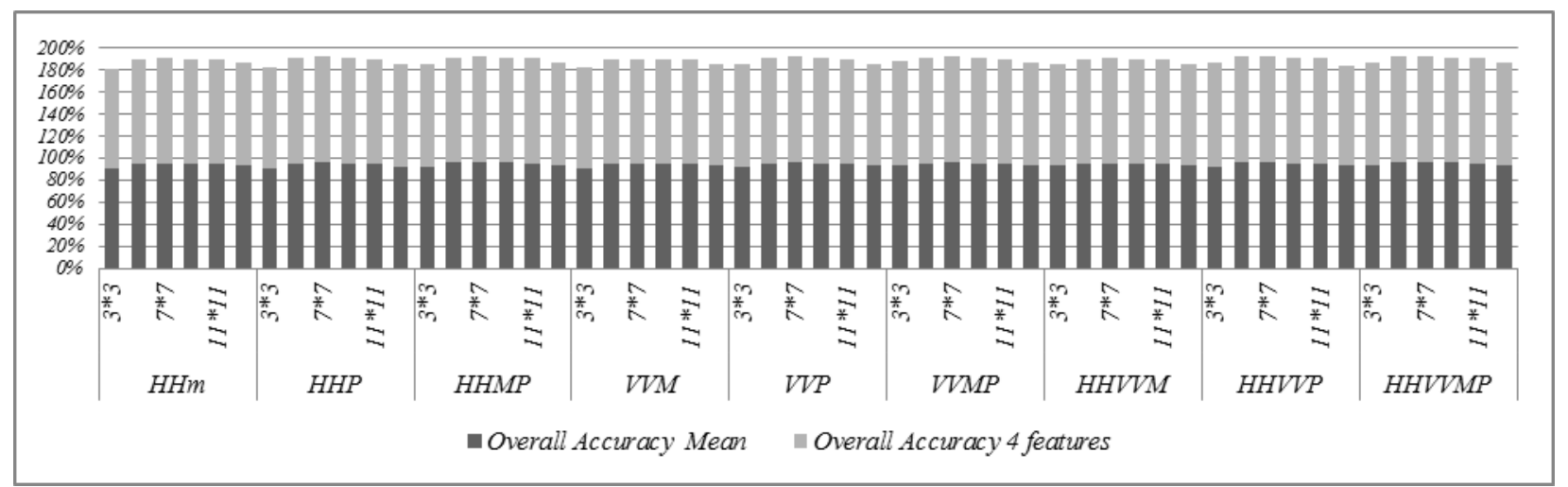

Figure 9. Overall accuracy of the SVM classification of Mean and combination of 4 features (Mean, Variance, Contrast and Dissimilarity) of the magnitude and power measurements Of $\mathrm{HH}$ and VV channels

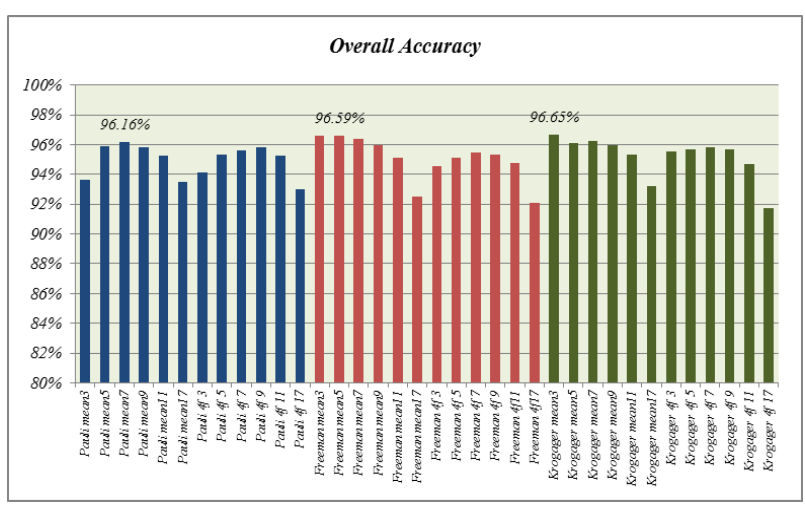

Figure 10. Overall accuracy of the SVM classification of Mean and combination of 4 features of Mean, Variance, Contrast and Dissimilarity of The Pauli, Krogager and Freeman decompositions.

\subsection{Discussion}

Each of the single polarimetric and fully polarimetric SAR data has their advantages in oil spill detection. The advantage of single polarimetric SAR data is usually its large swath coverage, while the advantage of fully polarimetric SAR data is the extra information contained in the measured scattering matrices, allowing discriminating of oil spills from the ocean background in a physical domain (Liu et al., 2011). A more advantage of fully polarimetric SAR data is extraction ability of decompositions that the single polarimetric SAR data do not have this capability. As a result, oil spill detection with fully polarimetric SAR is simpler, faster and more accurate.

As oil spills were not visually identified in $\mathrm{HV}$ and $\mathrm{VH}$ polarizations, magnitude and power measurements of $\mathrm{HH}$ and VV polarizations are extracted and merely used in the process in this paper.

Accuracy assessment measures demonstrate that in first step, power measurement of $\mathrm{VV}$ polarization is suitable for oil spill detection than the magnitude and power measures of $\mathrm{HH}$ and magnitude of VV polarization. The high sea reflectivity is due to Bragg scattering from capillary and short gravity waves. For that reason, the backscatter intensity in $\mathrm{VV}$ is stronger than
$\mathrm{HH}$ that in this research is confirmed. In the studies of (Fortuny-Guasch, 2003; Girard-Ardhuin and et al., 2003) this result is also obtained.

Krogager and Freeman decompositions present better results than the magnitude and power measurements of $\mathrm{HH}$ and $\mathrm{VV}$ polarizations and Pauli decomposition that is probably due to in Krogager and Freeman decompositions, neighbourhood pixels are also taken into account. In the last step, mean of Krogager decomposition has higher efficiency in detection of oil spills.

\section{CONCLUSION}

In this research a method for oil spill detection from full polarimetric SAR data is presented that it is based on SVM classification. Texture analysis and decomposition methods are also used in various steps of the algorithm. Also in this research, effect of The Pauli, Krogager and Freeman decompositions in oil spill detection is investigated. The results indicate that the Krogager decomposition is more efficient. The texture analysis is performed on magnitude and power images of $\mathrm{HH}$ and $\mathrm{VV}$ polarizations and the Pauli, Krogager and Freeman decompositions and sensitivity of the different window sizes are analysed. The results show that mean feature has better performance. This is because SAR images are inherently noisy and utilising this feature causes reduction of noise of the image. The results of sensitivity analysis of window sizes indicated window size of $5 \times 5$ pixels for Magnitude and power images and Freeman decomposition is best and, window size $7 \times 7$ pixels and $3 \times 3$ pixels for The Pauli, Krogager decompositions respectively have the best performance. Comparing of the final results with ground truth data indicated that the proposed algorithm has good results.

\section{References}

Brekke, C., Solberg, A., 2005. Oil spill detection by satellite remote sensing. Remote Sens. Environ. 95, pp. 1-13.

Cloude, S. and Pottier, E., 1996, A review of target decomposition theorems in radar polarimetry. IEEE Trans. Geosci. Remote Sens., 34(2), pp. 498-518.

Del Frate, F., Petrocchi, A., Lichtenegger, J., Calabresi, G., 2000. Neural networks for oil spill detection using ERS-SAR data. IEEE Transactions on Geoscience and Remote Sensing 38 (5), 2282-2287. 
Fingas, M. and Brown, C., 1997. Review of oil spill remote sensing, Spill Sci. Techn. Bull., 4(4), pp. 199-208.

Fingas, M., and Brown, C. E., (2011). Oil spill remote sensing: A review. Oil spill science and technology. Gulf Professional Publishing, Boston, pp. 111-169.

Fiscella, B., Giancaspro, A., Nirchio, F., Trivero, P., 2000. Oil spill detection using marine SAR images. International Journal of Remote Sensing 21 (18), 3561-3566.

Fortuny-Guasch, J., 2003. Improved oil slick detection and classification with polarimetric SAR, in Proc. POLin-SAR, Frascati, Italy. pp. 27.1.

Freeman, A., and Durden, S., 1992. A three component scattering model to describe polarimetric SAR data. Radar Polarimetry, SPIE- 1748, pp. 213-225.

Gade, M., Alpers, W., Huhnerfoss, H., Masuko, H. and Kobayashi, T., 1998. Imaging of biogenic and anthropogenic ocean surface films by the multifrequency/multipolarization SIR-C/X-SAR, J. Geophys. Res., 103(C9), pp. 18851-18866.

Girard-Ardhuin, F., Mercier, G., and Garello, R., 2003. Oil slick detection by SAR imagery: potential and limitation, Proc. OCEANS 2003, 1, pp. 164-169.

Holt, B., (2004). SAR imaging of the ocean surface. In C. R. Jackson, \& J. R. Apel (Eds.), Synthetic aperture radar (SAR) marine user's manual. Washington DC: NOAA NESDIS Office of Research and Applications. pp. 25-79.

Jones, C., Minchew, B., and Holt, B., 2011. Polarimetric decomposition analysis of the Deepwater Horizon oil slick using L-band UAVSAR data. IGARSS, Vancouver, BC, Canada, pp. 2278-2281.

Karathanassi, V., Topouzelis, K., Pavlakis, P., Rokos, D., 2006. An object-oriented methodology to detect oil spills. International Journal of Remote Sensing 27 (23), 5235-5251.

Krogager, E., 1990. A new decomposition of the radar target scattering matrix. Electron. Lett, 26(18), pp. 1525-1526.

Liu, P., Li, X., Qu, J. J.,Wang, W., Zhao, C. and Pichel, W. 2011. Oil spill detection with fully polarimetric UAVSAR data, Marine Pollution Bulletin, 62, 2611-2618.

Marghany, M., 2001. Radarsat automatic algorithms for detecting oil spill pollution, Int. J. of Applied Earth Observation and Geoinformation, 3 (2), pp. 191-196, 2001.

Migliaccio, M., Trangaglia, M., 2004. Oil spill observation by SAR: a review, US-Baltic International Symposium, Klaiped, Lithuania.

Migliaccio, M., Gambardella, A. and Tranfaglia, M., 2007. SAR polarimetry to observe oil spills, IEEE Trans. Geosci. Remote Sens., 45(2), pp. 506-511.

Migliaccio, M., Nunziata, F. and Gambardella, A., 2009. On the copolarised phase difference for oil spill observation, Int. J. Remote Sens., 30(6), pp. 1587-1602.

Pavlakis, P., Tarchi, D., Sieber, A., 2001. On the Monitoring of Illicit Vessel Discharges, A reconnaissance study in the Mediterranean Sea, European Commission, EUR., 19906 EN.

Schuler, D., Lee, J. and De Grandi, G., 2004. Spiral eddy detection using surfactant slick patterns and polarimetric SAR image decomposition techniques, in Proc. IGARSS, Anchorage, AK, 1, pp. 212-215.
Stathakis, D., Topouzelis, K., Karathanassi, V., 2006. Largescale feature selection using evolved neural networks, In Proceedings of SPIE, Image and Signal Processing for Remote Sensing XII, Bruzzone (Ed.), 6365.

Topouzelis, K., Karathanassi, V., Pavlakis, P., Rokos, D., 2003. Oil Spill Detection: SAR Multi-scale Segmentation \& Object Features Evaluation. In Proceedings of SPIE, Remote Sensing of the Ocean and Sea ice 2002, 23-27 September, Creta, Greece, Bostater and Santoleri (Ed.), 4880, 77-87.

Topouzelis, K., 2008. Oil spill detection by SAR Images: dark formation detection, feature extraction and classification algorithms. Sensors 8(10), pp. 6642-6659.

Topouzelis, K., Stathakis, D., Karathanassi, V., 2009. Investigation of genetic algorithms contribution to feature selection for oil spill detection. International Journal of Remote Sensing 30 (3), 611-625.

Velotto, D., Migliaccio, M., Nunziata, F., and Lehner S., 2010. Oil-slick observation using single look complex TerraSAR-X dual-polarized data. Int. Geosci. Remote Sens. Symp, pp. 36843687.

Zhang, B., Perrie, W., Li, X., Pichel, W., 2011. Mapping sea surface oil slicks using RADARSAT-2 quad-polarization SAR image, Geophysical Research Lettersdoi, 38(10). 